\title{
The Agger Nasi cell and uncinate process, the keys to proper access to the nasolacrimal drainage system*
}

\author{
M.B. Soyka ${ }^{1}$, T. Treumann ${ }^{1}$, C.Th. Schlegel ${ }^{1}$ \\ 1 Department Otolaryngology Head and Neck Surgery, Luzerner Kantonsspital, Lucerne, Switzerland \\ 2 Department Radiology, Luzerner Kantonsspital, Lucerne, Switzerland
}

\begin{abstract}
SUMMARY Background: Profound knowledge of the surgical anatomy is required to perform any dacryocysto-rhinostomy (DCR) procedure. In endoscopic endonasal DCR surgery, the uncinate process (UP) and/or agger nasi (AN) cell are frequently found to obstruct access to the lacrimal fossa. The aim of this study was to analyse the radiological anatomy of the UP and the AN cell in relation to the lacrimal sac.
\end{abstract}

Methods: Preoperative computed tomography (CT) scans of patients undergoing DCR and other endoscopic sinus surgeries were analysed regarding their anatomical variations concerning the UP and the AN cell at the level of the common canaliculus. The covering of the lacrimal fossa by the UP was graded and a surgically relevant AN cell was sought after.

Results: CT datasets of 60 sides were included. Analysis showed an AN cell overlying the upper parts of the lacrimal sac in 55\% of patients. An anteriorly attaching UP covering at least 50\% of the lacrimal fossa was found in 63\% of individuals.

Conclusion: In this study more than 80\% of all patients presented with either a very anteriorly attached UP or an AN cell that would have to be removed to perform effective endoscopic DCR. The AN cell and the UP need to be included in the endonasal surgical concept.

Key words: anatomy, dacryocystorhinostomy, endoscopic surgical procedure, ethmoid sinus, ethmoid bone

\section{INTRODUCTION}

The nasolacrimal sac and duct are the target points for both endonasal and external approaches for dacryocysto-rhinostomy (DCR) procedures. The knowledge of the quite demanding anatomy of the lacrimal fossa (LF) as well as its surroundings is of utmost importance for any lacrimal surgeon. The borders of the nasolacrimal system include the anterior ethmoidal air cells, the frontal process of the maxilla, the inferior turbinate and the lacrimal bone itself.

Lately, it has been proposed that successful DCR can only be achieved by the proper exposure of the proximal parts of the lacrimal sac up to the common canaliculus and fundus ${ }^{(1,2)}$. Whilst the endonasal approach to the lacrimal sac was described by the removal of the frontal process of the maxilla and lacrimal bone in most of the standard textbooks, later studies have taken a closer look at the exact position of the tear sac within the lateral nasal wall in relation to the middle turbinate ${ }^{(1)}$ and the specific structures overlying the drainage pathway ${ }^{(3)}$. Special interest was focused upon the uncinate process (UP) ${ }^{(4)}$.
At our institution, we perform endoscopic DCR at the level of the common canaliculus and thus very proximally. In our experience, it is not only the UP that often needs to be removed prior to reaching the frontal process of the maxilla and lacrimal bone. An agger nasi (AN) air-cell is frequently found intraoperatively situated medially to the lacrimal sac. This cell needs to be removed to gain appropriate access. As there is a close relationship between the UP and AN, it is important to examine and analyse these structures as one unit ${ }^{(5)}$. We conducted this study to verify these clinical findings in preoperative CT images of patients with and without tear sac pathology.

\section{MATERIALS AND METHODS}

Study design/CT scan

A retrospective radiological case series study was performed using the electronic PACS system at our institution. Over a period from $10 / 2003$ to $04 / 2009$, all consecutive preoperative CT scans of patients operated for DCR, were retrieved. To increase the case load, we also included routine preoperative CT scans from patients undergoing endoscopic sinus surgery 
from $03 / 2007$ and $12 / 2008$ at the same institution. All CTs were carried out on a Siemens Somatom Definition CT Scanner (Siemens Medical Systems, Erlangen, Germany) without contrast agent. Slice thickness was between 0.6 to $0.8 \mathrm{~mm}$. Until June 2008, CTs were performed with a maximum tube current of 400 mAs. From July 2008, all CTs were performed with a low dose protocol with a maximum tube current of 80 mAs. Tube voltage was $120 \mathrm{kV}$. Soft tissue (H31S kernel) and high resolution bone datasets $(\mathrm{H} 70 \mathrm{H}$ kernel) were calculated. The datasets were analysed by multiplanar reformats (MPR) in adapted imaging planes by both the first (M.S.) and the senior (C.S.) author independently. Differences were eliminated in a second interpretation session in consensus.

\section{Patient selection}

In 108 patients, 120 sides were treated by endonasal DCR. Out of these, 26 patients (24\%) with preoperative CT scans were available for $30(25 \%)$ planned DCR procedures. CT-scanning was not routinely performed before DCR. In DCR patients, only the operated side(s) were chosen for evaluation, whereas in the other subjects only one side was evaluated randomly according to the last digit of the year of birth. If the year was even, the right side was analysed, in odd birth-years, the left hand side was evaluated.

Exclusion criteria were: previous surgery on the same side; gross pathology of the paranasal sinuses that would influence the position or appearance of important landmarks such as the UP, AN or the lacrimal system; and age $<16$ years.

\section{Assessment and analysis}

We looked for the presence of an AN cell covering the lacrimal sac at the location of the common canaliculus (Figure 1).

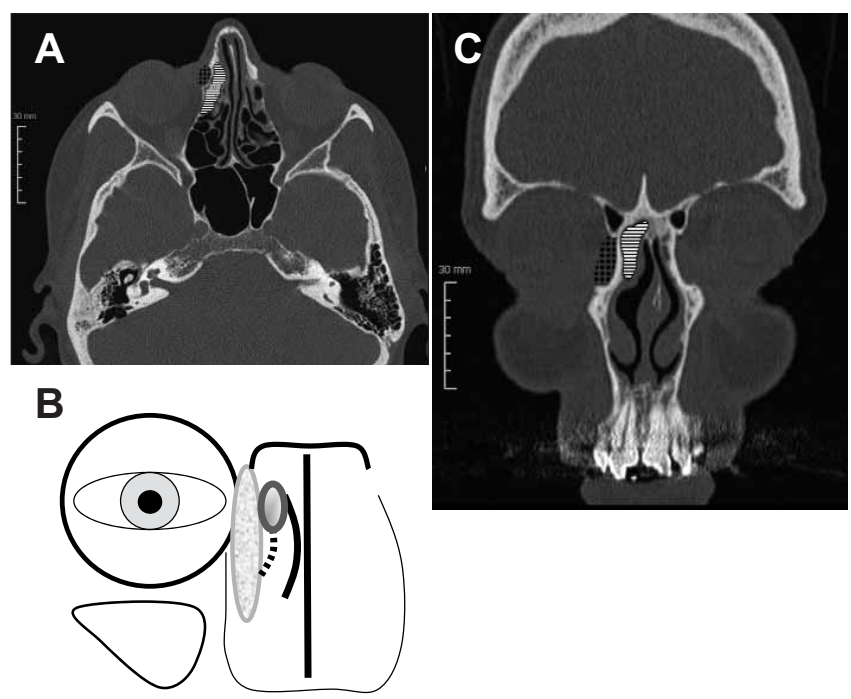

Figure 1. Agger Nasi cell (blue) covering the lacrimal sac (red).

A) Axial CT at the level of the common canaliculus $(5 \mathrm{~mm}$ below the fundus of the lacrimal). B) Schematic drawing of the lacrimal fossa and the adjacent structures. C) Coronal CT Scan of the same patient.
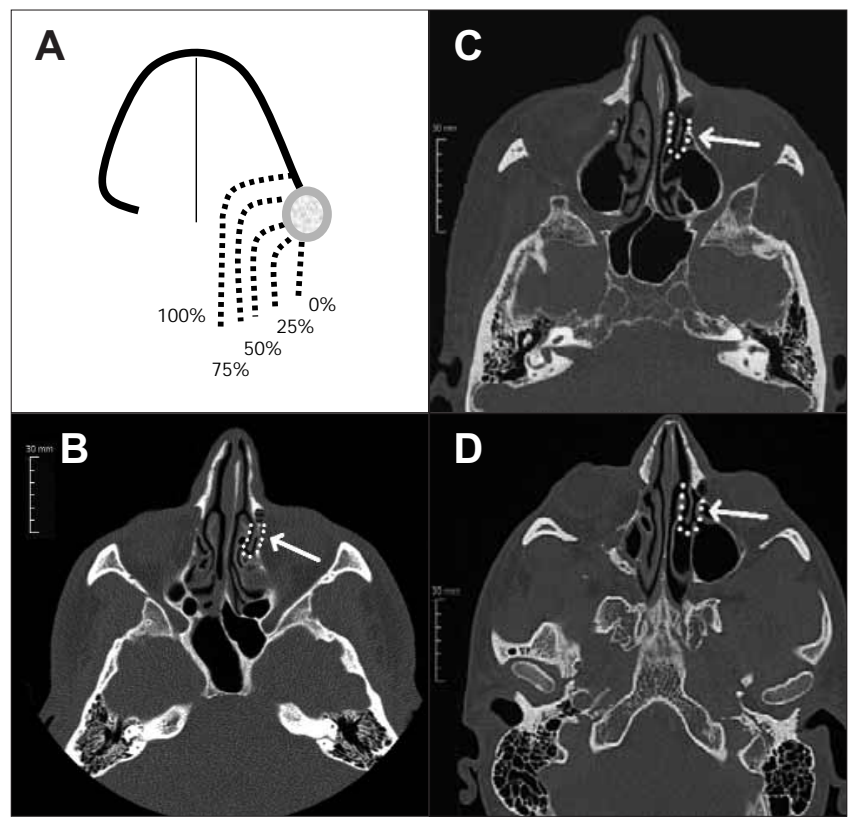

Figures 2. Axial CT-scans of three different patients.

A) Schematic illustration of the attachment of the UP. B) The UP (green) does not cover the lacrimal drainage system (red) at all. C) shows $25 \%$ coverage. D) The lacrimal sac (red) is fully covered by the UP (green). Position at the common canaliculus ( $5 \mathrm{~mm}$ below the fundus of the lacrimal sac).

According to Wormald and colleagues, this position is located $5 \mathrm{~mm}$ below the fundus of the $\mathrm{sac}^{(1)}$. We therefore scrutinised the $\mathrm{CT}$ datasets in a range of $4 \mathrm{~mm}$ above and below this position for the existence of such a covering cell in the axial, coronal and sagittal planes.

Secondly, the insertion and covering of the UP over the lacrimal sac at the same position $(5 \mathrm{~mm} \pm 4 \mathrm{~mm}$ below the fundus) was assessed and graded with a 5 step grading system from $0 \%$ (not covering at all) up to $100 \%$ (fully covering), in steps of 25\% (Figure 2).

\section{Statistics}

Data was stored in a Microsoft Excel (Microsoft Deutschland $\mathrm{GmbH}$, Germany) spreadsheet and analysed statistically using the SPSS program (Version 17, SPSS inc., Chicago, IL, USA). Descriptive statistics were performed for the parameters: AN cell covering yes/no; UP covering 0-100\%.

\section{RESULTS}

Altogether 60 sides were assessed. Thirty applicable images in the "DCR-group" were found in 26 patients. Additional 30 sides in 30 different individuals were available from other

Table 1. Proportions for presence of an AN cell obstructing access to the lacrimal sac.

\begin{tabular}{cc}
\hline $\begin{array}{l}\text { Agger Nasi Cell overlying } \\
\text { Lacrimal Sac }\end{array}$ & Patients \\
\hline yes & $33(55 \%)$ \\
no & $27(45 \%)$ \\
\hline
\end{tabular}


Table 2. Distribution of type of attachment of the UP in relation to the lacrimal fossa at the level of the common canaliculus.

\begin{tabular}{cc}
\hline $\begin{array}{c}\text { Uncinate Process } \\
\text { Attachment }\end{array}$ & Patients \\
\hline $0 \%$ & $12(20 \%)$ \\
$25 \%$ & $10(17 \%)$ \\
$50 \%$ & $15(25 \%)$ \\
$75 \%$ & $6(10 \%)$ \\
$100 \%$ & $17(28 \%)$ \\
\hline
\end{tabular}

paranasal surgical procedures. Median age in the "DCR-group" and "ESS-group" was 60 and 35 years, respectively. Male to female ratio was 4:26 in the "DCR-group" and 17:13 in the "ESS-group". Indications for surgery in the latter patients mainly consisted of chronic rhinosinusitis with and without polyps [22]; other reasons were mycetoma [2], pyocoele, mucocoele, inverted papilloma, choanal atresia, approach to pituitary gland and pseudotumor of the orbit. None of the underlying pathologies had direct or indirect contact to the evaluated nasolacrimal system. The presence of an AN-Cell overlying the lacrimal sac was found in 55\% (Table 1).

The analytical results for the upper parts of the lacrimal sac being covered by the UP are listed in Table 2. A wide variation of the anterior attachment can be appreciated. The region of the common canaliculus was covered by more than a half in $63 \%$ of all individuals.

There was no inter-observer difference concerning an overlying AN cell. There were four cases of divergent interpretation of the coverage of the lacrimal sac by the UP. These differences were eliminated by consensus.

\section{DISCUSSION}

Thorough knowledge of the anatomy remains the key to any

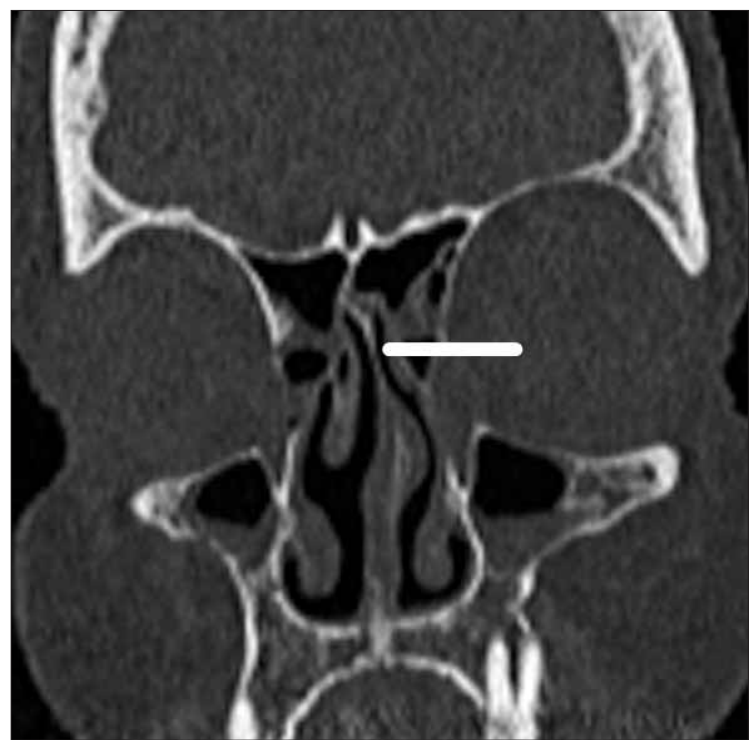

Figure 3. Identification of the fundus of the lacrimal sac using bony landmarks on a coronal scan. surgical procedure. Not much published information however exists about the bony structures of the lateral nasal wall adjacent to the lacrimal drainage system ${ }^{(6)}$. Most of the detailed data concentrates on lacrimal duct injuries during functional endoscopic sinus surgery rather than on DCR procedures themselves ${ }^{(7,8)}$. The relation of the AN and UP to the nasolacrimal system has not even been appreciated in standard textbooks of the nasal anatomy ${ }^{(9)}$.

It is the common belief in current literature that long lasting positive results depend on the extent and location of the rhinostomy in DCR ${ }^{(2,10)}$. Proper exposure, marsupialisation and integration of the lacrimal sac into the lateral nasal wall is only possible at the level of the common canaliculus. Wormald et al. first described the exact location of the lacrimal sac in computed tomography and clearly defined the distance of the common canaliculus from the fundus of the lacrimal sac ${ }^{(1)}$. We could easily measure this distance by identifying the fundus on coronal scans and correlating this point with axial images using MPR (Figure 3). We hereby avoided underestimating the variance caused by the curvilinear shape of the UP.

The lacrimal sac is mostly described to be located anterior to the UP and middle turbinate, being covered anteriorly by thick bone of the frontal process of the maxilla and a thin layer of lacrimal bone. In our study, we could show a wide variation of the position of the uncinate process. It ranged from a far anterior insertion at the AN with full coverage of the tear sac (Figure 4) to a far posterior location at the lacrimal bone where it does not obstruct access in surgery. This was also appreciated by Fayet et al. who considered a "Systematic Unciformectomy for standard DCR" to be necessary to gain entry to the $\mathrm{LF}^{(4)}$. In contrast to his descriptions, we prefer a more differentiated percental grading of the attachment of the UP. The anterior insertion was also recognised in one case

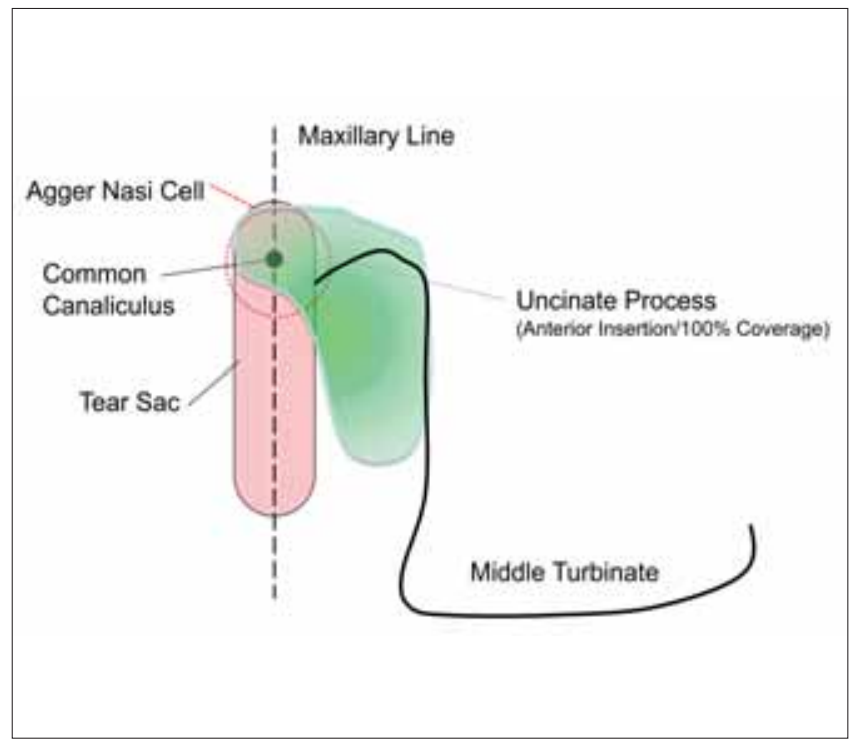

Figure 4. View of the lateral nasal wall in the region of the lacrimal system, with overlying AN cell and a fully covering uncinate process. 
report, where the anatomical variation of a thick UP was present ${ }^{(11)}$. With the results of our study, we strongly support Fayet's conclusion as more than $60 \%$ of the patients showed an "obstructing" UP of not less then 50\% of the LF. According to our data however, taking away the uncinate process as a standard procedure is not always necessary. Sometimes a partial removal is sufficient and in some cases no resection is required at all. Our results however refer only to the location at the common canaliculus, where we and other authors recommend DCR should be performed at ${ }^{(2)}$. As mentioned before, we have to appreciate the curvilinear shape of the UP (as shown in Figure 4) and therefore this structure might not be an obstacle for other surgeons who perform DCR at a different level, i.e. the nasolacrimal duct.

The incidence of an AN cell in general is $80 \%-89 \%{ }^{(12,13)}$. If the AN cell is located at the level of the upper part of the lacrimal sac, it may obstruct a surgeon's way towards the LF or can be mistaken for the lacrimal sac. This was not appreciated sufficiently in the past literature. There are some ancient anatomical descriptions ${ }^{(14,15)}$. The latest publication dates back to the early nineties ${ }^{(16)}$. It alludes to the close relation of the anterior ethmoid and found in more than $90 \%$ of the patients undergoing external DCR an ethmoidal air cell anterior to the posterior lacrimal crest. In our series, an AN cell could be found in 55\% of the individuals at a level that could be troublesome for the endoscopic surgeon.

Patients with and without lacrimal sac pathologies were included in this study. We have no reason to believe that anatomy should be any different in regard to the uncinate process or agger nasi between patients with sino-nasal pathology patients undergoing a DCR procedure. Patients not undergoing DCR were carefully selected not to have disease in proximity to the lacrimal system.

A female preponderance in the DCR groups was found similarly to other reports in the literature ${ }^{(17)}$.

In analogy to Professor Wormald's legendary description of the AN cell being the key to understanding frontal sinus drainage pathways ${ }^{(18)}$, we believe that the AN cell and uncinate process together form the key to proper understanding of the anatomy of the lacrimal fossa, as well. We recommend the use of computed tomographies for surgeons who are not familiar with the endonasal anatomy in this area to appreciate the variability of the structures medial to the lacrimal sac.

\section{CONCLUSION}

We conclude that the uncinate process and the AN air-cells form a key point for a successful endonasal approach to the lacrimal sac. In addition to the removal of parts of the frontal process of the maxilla and the lacrimal bone, in most cases either an overlying AN cell or a covering UP needs to be removed before gaining access to the lacrimal and maxillary bone in DCR. These structures also need to be considered in other surgical concepts such as the external DCR approach. In the modified Toti operation, the lacrimal pathway is also drained at the level of the common canaliculus into the nose, where both structures can be encountered.

\section{REFERENCES}

1. Wormald P, Kew J, Van Hasselt A. Intranasal anatomy of the nasolacrimal sac in endoscopic dacryocystorhinostomy. Otolaryngol Head Neck Surg 2000; 123: 307-310.

2. Tsirbas A, Wormald P. Mechanical endonasal dacryocystorhinostomy with mucosal flaps. Otolaryngol Clin North Am 2006; 39: 1019-36, viii.

3. Fayet B, Racy E, Assouline M, et al. Surgical anatomy of the lacrimal fossa a prospective computed tomodensitometry scan analysis. Ophthalmology 2005; 112: 1119-1128.

4. Fayet B, Racy E, Assouline M. Systematic unciformectomy for a standardized endonasal dacryocystorhinostomy. Ophthalmology 2002; 109: 530-536.

5. Zhang L, Han D, Ge W, et al. Anatomical and computed tomographic analysis of the interaction between the uncinate process and the agger nasi cell. Acta Otolaryngol 2006; 126: 845-852.

6. Rebeiz E, Shapshay S, Bowlds J, et al. Anatomic guidelines for dacryocystorhinostomy. Laryngoscope 1992; 102: 1181-1184.

7. Yung M, Logan B. The anatomy of the lacrimal bone at the lateral wall of the nose: its significance to the lacrimal surgeon. Clin Otolaryngol Allied Sci 1999; 24: 262-265.

8. Unlü H, Gövsa F, Mutlu C, et al. Anatomical guidelines for intranasal surgery of the lacrimal drainage system. Rhinology 1997; 35: 11-15.

9. Lang J. Clinical Anatomy of the Nose, Nasal Cavity, and Paranasal Sinuses: George Thieme; 1989.

10. Tsirbas A. Lacrimal fossa anatomy. Ophthalmology 2006; 113: 1475-1476; author reply 6-7.

11. Mehta A, Puri N. An unusual variation in the anatomy of the uncinate process in external dacryocystorhinostomy. Indian $\mathrm{J}$ Ophthalmol 2008; 56: 413-416.

12. Lee W, Kuhn F, Citardi M. 3D computed tomographic analysis of frontal recess anatomy in patients without frontal sinusitis. Otolaryngol Head Neck Surg 2004; 131: 164-173.

13. Leunig A, Betz C, Sommer B, et al. Anatomic variations of the sinuses; multiplanar CT-analysis in 641 patients. Laryngorhinootologie 2008; 87: 482-489.

14. Whitnall S. The relations of the lacrimal fossa to the ethmoidal cells. In: Ophthalmic Rev; 1911:321-5.

15. Cohen A, Mercandetti M, Brazzo B. The Lacrimal System, Diagnosis, Treatment, and Surgery: Springer; 2006.

16. Blaylock W, Moore C, Linberg J. Anterior ethmoid anatomy facilitates dacryocystorhinostomy. Arch Ophthalmol 1990; 108: 17741777.

17. Duffy M. Advances in lacrimal surgery. Curr Opin Ophthalmol 2000; 11: 352-356.

18. Wormald P. The agger nasi cell: the key to understanding the anatomy of the frontal recess. Otolaryngol Head Neck Surg 2003; 129: $497-507$

\author{
Michael Soyka, MD \\ Dept. Otolaryngology Head and Neck Surgery \\ University Hospital \\ CH-8091 Zurich \\ Switzerland
}

Tel: +41-44-255 1111

Fax: +41-44-255 4556

E-mail: michael@soyka.ch 